\title{
Integration of real-intelligence in energy management systems to enable holistic demand response optimization in buildings and districts
}

\author{
Ander Romero, Pablo de Agustín \\ Sustainable Construction Division \\ Tecnalia Research \& Innovation \\ Derio, Spain \\ ander.romero@tecnalia.com,pablo.deagustin@tecnalia.com
}

\author{
Tasos Tsitsanis \\ IT \& Innovation Unit \\ Hypertech S.A. \\ Athens, Greece \\ t.tsitsanis@hypertech.gr
}

\begin{abstract}
Although multiple trials have been conducted demonstrating that demand side flexibility works and even though technology roll-out progresses significantly fast, the business application of residential and small tertiary demand response has been slow to develop. This paper introduces a holistic demand response optimization framework that enables significant energy costs reduction at the consumer side, while introducing buildings as a major contributor to energy networks' stability in response to network constraints and conditions. The backbone of the solution consists in a modular interoperability and data management framework that enables open standards-based communication along the demand response value chain. The solution is validated in four large-scale pilot sites, incorporating diverse building types, heterogeneous home, building and district energy systems and devices, a variety of energy carriers and spanning diverse climatic conditions, demographic and cultural characteristics.
\end{abstract}

Keywords—energy efficiency; demand response; interoperability

\section{INTRODUCTION}

Since the liberalisation of the energy sector in Europe, significant developments have taken place, including growing competition and market integration, decreasing wholesale prices, and the enhanced uptake of renewable energy sources. Nevertheless and even though consumer empowerment has become a buzzword in European energy policy, options and benefits for consumers have so far remained limited [1]. While possibilities for larger commercial and industrial consumers have started to develop and active consumer engagement in demand response programmes is gaining in relevance, this is not the case today for most residential and medium/ small tertiary consumers. Commercial and industrial demand response is technically and economically viable now, however, residential and small tertiary resources are still excluded from the market.

In fact, buildings comprise a huge source of flexible energy demand and storage, providing distribution and transmission system operators with the services they need to balance available supply and manage power quality at all times. So far there are only few Member States around the European Union that have mandated the participation of energy consumers in demand response [2], especially focusing on implicit demand response

This paper is part of a project that has received funding from the European Union's Horizon 2020 research and innovation programme under grant agreement No 768614. This paper reflects only the author's views and neither the Agency nor the Commission are responsible for any use that may be made of the information contained therein. schemes, but still prices remain regulated (and the offering unattractive), not allowing small consumers to monetize in practice their flexibility, enhance comfort and maximize cost savings [1] [3] [4].

Even if the de-facto commitment stated in the recent communication for completing the internal energy market [5], which is further supported with the entry into force of the Winter Package [6], manages to successfully tackle all major regulatory barriers for demand response and accelerate its full introduction in national markets, there still remain several key enablers, i.e. consumer empowerment, end-to-end interoperability between energy networks, building energy management systems (BEMS) and devices, etc., that need to be satisfied towards unleashing the huge potential and enhancing the commercial viability of demand side flexibility offered by the building sector, while maximizing its value for both prosumers and energy market stakeholders.

Integration of demand response in BEMS and sub-sequent penetration in energy markets heavily relies on the deep and comprehensive understanding of real-life complexities imposed during actual operation, as well as, on the smooth end-to-end interoperable communication between all actors involved. Both these parameters span four interrelated areas: physical systems (buildings, their equipment and their usage, along with districts and energy networks), human systems (occupants and their behaviours), energy markets (energy tariffs, transaction requirements and access of small consumers) and the general surrounding environment (weather fluctuations and impact on the other systems).

The inadequacy of current technical offerings, business practices, market structures and regulatory frameworks to effectively address and smoothly integrate all these interrelated domains is the root cause for the slow pace of demand response introduction in energy markets and the failure to empower consumers towards becoming more active energy market actors.

\section{CONCEPTUAL SOLUTION}

In response to the aforementioned challenges and needs this paper presents HOLISDER project which introduces a holistic demand response optimization framework that enables

(C) 2018 IEEE. Personal use of this material is permitted. Permission from IEEE must be obtained for all other uses, in any current or future media, including reprinting/republishing this material for advertising or promotional purposes, creating new collective works, for resale or redistribution to servers or lists, or reuse of any copyrighted component of this work in other works. Integration of real-intelligence in energy management systems to enable holistic demand response optimization in buildings and districts. This is the author's version of the article the final version of record is available at DOI: 10.1109/EEEIC.2018.8494522 . 
significant energy costs reduction at the building/consumer side, while introducing small and medium sized buildings (residential and non-residential ones) as a major contributor to energy networks' stability through optimized energy management in response to network constraints and conditions.

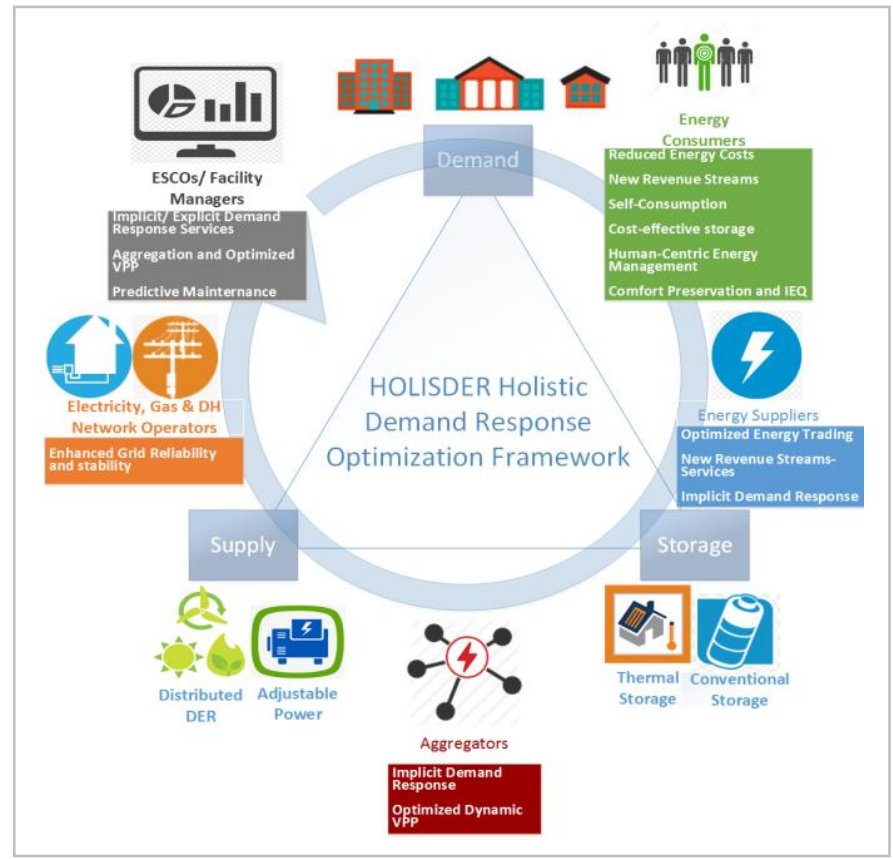

Fig. 1. Actors involved in the holistic demand response optimization framework

For the successful deployment of demand response in European Union markets and focusing on the key enablers for the realization of this high-level target, the solution enables: (1) significant energy costs savings for energy consumers, (2) creation of new revenue streams for energy consumers and their introduction as active balancing and ancillary assets in energy markets, (3) wide promotion of self-consumption for prosumers, (4) utilization of the currently unleashed storage capacity of buildings, (5) proper tackling of consumers' reluctance to participate in demand response, (6) further facilitation of consumers' participation in energy markets, (7) high replicability across different building types and systems, (8) advanced adaptability to demand response regulations around European Union Member States, and (9) enhanced operational stability and security of energy networks.

The backbone of the solution consists in an open and modular end-to-end interoperability and data management framework that enables open standards-based communication along the demand response value chain. In more detail, the interoperability and secure data management framework couples two major technologies/products to ensure seamless integration, communication and operation on top of any building and district energy management system, as well as, smart home systems and devices.

Implicit demand response stands at the forefront of the demand response optimization framework. Dynamic and realtime energy tariff schemes are available to consumers towards altering their energy consumption patterns and shifting them away from peak periods.

Exposure of consumers in real-time energy prices stands as the first action of empowering and engaging them in energy market transactions. To ensure enhanced and long-lasting engagement and facilitate consumers' participation in the market, a series of innovative solutions and applications are introduced, to enable: (1) personalized informative billing, (2) human-centric energy management and control decision support, (3) scheduling and automation, (4) self-consumption promotion and cost-effective storage, and (5) predictive maintenance advising.

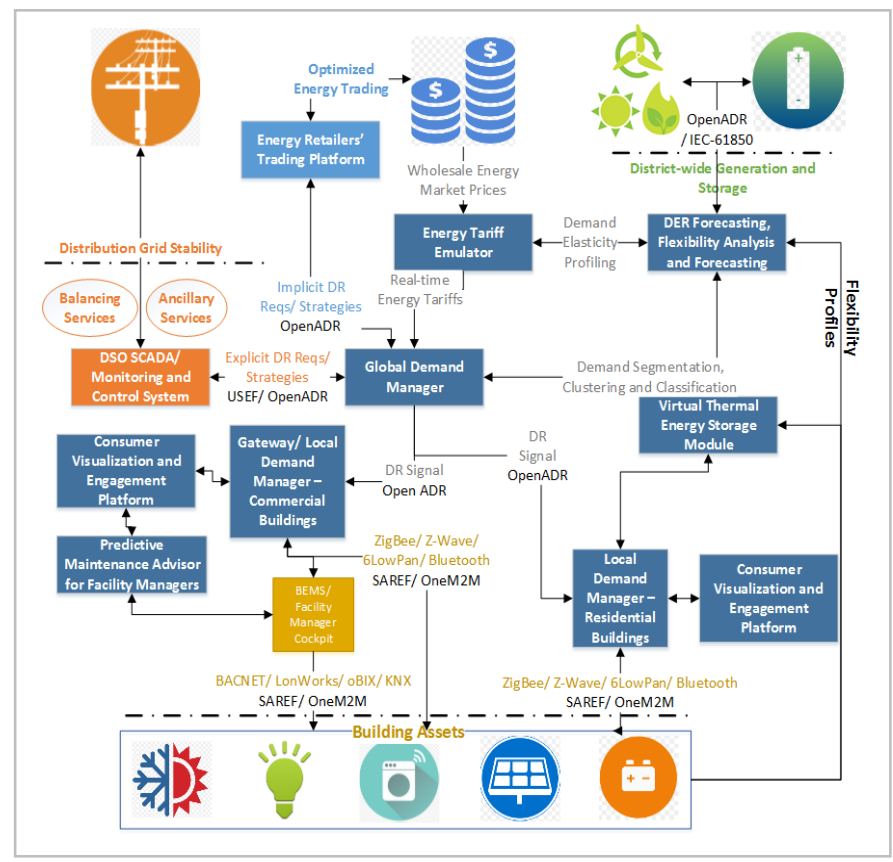

Fig. 2. Detailed representation of the solution

\section{SYSTEM ARCHITECTURE}

The system architecture contains three high level functional constituents which are further broken down at different deployment levels to address all aspects introduced in the solution. These are:

- The integrated interoperability and secure data management framework, ensuring technical and semantic/ syntactic interoperability across the demand response value chain on the basis of widely adopted open standards.

- The demand response optimization and control dispatch decision support system (DSS) deployed both at the building and district level and incorporating a variety of functions to support optimal decision-making at both levels.

- The visualization platform and end-user toolkit components, referring to the front-end applications. 


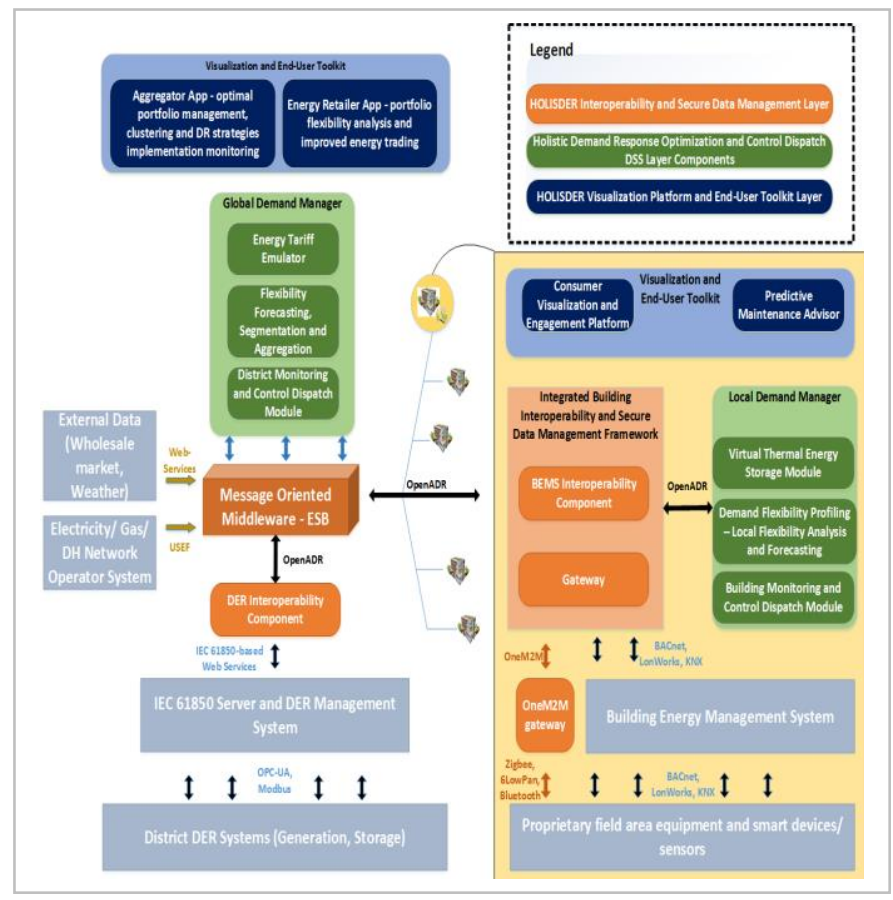

Fig. 3. Conceptual architecture of the solution

\section{A. Interoperability and secure data management layer}

The interoperability and secure data management layer integrates two main technological products, which are loosely coupled to enable easy adaptation in each different building type:

- An OpenADR 2.0b enabled component which ensures standardized multi-protocol communication and data exchange with the majority of commercially available BEMS/ protocols (BACnet, KNX, LonWorks, OPC-UA, Modbus) and distributed energy resources (DER) management systems (IEC 61850-based web communication).

- A gateway which comprises the basis upon which further extensions and enhancements are performed towards configuring a M/490-based integrated smart home gateway/ energy management system (EMS) module, enabling end-to-end interoperable communication between the global demand manager and individual smart home devices and sensors (compatibility with OneM2M standard and further extension to support mapping of OneM2M to proprietary smart home communication protocols, e.g. Zigbee, BLE, 6LowPan, etc.).

A message oriented middleware (ESB) is deployed at the district level and enables fine-grained and interoperable communication and data exchange with a variety of heterogeneous sources (buildings, district DER management systems, demand response actors' proprietary systems, weather data sources and wholesale energy prices). The ESB comprises in an open platform and application software framework that establishes seamless, transparent and homogeneous standardsbased (OpenADR 2.0b, USEF) interfaces to all integrated components. Furthermore, it provides appropriately defined semantic virtual entities (semantically enhanced components) thus incorporating the necessary semantics for the efficient management of the various information streams.

To facilitate end-to-end interoperability and information exchange between all components involved in the demand response value chain, these solutions are integrated into a unified interoperability and data management framework, to ensure interoperability (physical, communication, semantic and functional) with any type of EMS and smart home device in a modular manner, according to the building type and available systems. This allows their utilization either in an integrated framework in BEMS-equipped "smart" non-residential buildings, or individually, in BEMS-equipped non-residential buildings and smart homes on the basis of widely accepted open standards.

To address requirements for the definition of a single smart home interface and protocol, the gateway is appropriately extended to comply with OneM2M (incorporating the recently released SAREF ontology) and OpenADR 2.0b to ensure seamless integration and end-to-end communication between the demand response optimization framework and any type of flexibility source (device) residing in different types of buildings (both OneM2M-enabled, but also existing ones enabling communication over proprietary protocols). The aforementioned extensions and adoption of open standards guarantees the realization of an open and end-to-end interoperable framework, on the basis of a single communication and data exchange interface, even with existing smart home offerings and setups (not only focusing on future, OneM2M-enabled devices), to address the current weaknesses of OneM2M standard definition and enable effective tackling of a major barrier for the short-term commercialization of the solution.

With regards to the latter a gateway/ adapter is provided between OneM2M that pervades communications with the smart home devices and the OpenADR protocol. The solution abides by the provisions of the flexibility functional architecture for the smart home from the CEN-CENELEC-ETSI Coordination Group based on the M/490 mandate. Essentially the gateway hosts a virtual end node and perform bi-directional protocol and data-model translation in a semantically and syntactically coherent manner. The flexibility functional architecture already assumes smart home device compatibility with OneM2M which is not the case yet for available smart home devices. So the gateway also handles interoperability with the popular smart home protocols to bridge this gap. Data modelling activities are undertaken in order to generate the knowledge graph and support the necessary protocols as well as to interface with the OneM2M standard.

Semantic data enrichment is addressed in a novel common information model (CIM). The CIM enables the performance of semantic event processing, enrichment and annotation within the real-time requirements of the application components. For completeness purposes, the interoperability and data management framework includes functionality to bridge the communication gap with management systems of typical distributed energy resources, including district-level storage or 
generation devices. Even though most DERs already in the field are Modbus or OPC compatible, the IEC 61850 standard is gaining popularity for their monitoring and control. As a result, the interoperability and data management layer includes an OpenADR to IEC 61850 Gateway. OpenADR is also currently under standardization by the IEC as the IEC 62746 standards family. An existing open source IEC 61850 client implementation is used to interface with the DER management systems.

\section{B. Holistic demand response optimization and control dispatch DSS layer}

The holistic demand response and control dispatch DSS layer comprises in two main components that enable the establishment of a stepped optimization process, beginning with high level optimization considering aggregated volumes of flexibility at the district level and, subsequently, transferring the required flexibility amounts to the individual loads and assets residing in each building and defining instantiated load control strategies (to be applied manually or automatically), on the basis of appropriate demand response signals (implicit or explicit) communicated to the consumers.

In more detail, the current layer consists in the following distinct components:

- The global demand manager. This component is responsible for the definition of the high-level demand response strategies to be deployed over appropriately selected clusters of consumers. In implicit demand response programmes, the DSS utilizes and processes information received by the following sub-components to define appropriate consumer clusters that can effectively participate in demand response events, based on their defined flexibility against different levels of energy tariffs:

- Energy tariff emulator which is responsible for constantly collecting and analysing wholesale energy price data, renewables output, along with information about energy networks constraints, to produce real-time retail tariffs for each of the different energy carriers, such as electricity, district heating and gas, while considering special situations occurring in energy networks and addressing them in special tariff schemes such as critical peak pricing.

- Flexibility forecasting, segmentation and aggregation module which is responsible for the multidimensional analysis, correlation and efficient management of consumer profiles and consumer flexibility, along with response capacity to specific energy price signals. The module enables the dynamic segmentation of consumers' flexibility to volatile prices (considering also both spatial and temporal commonalities or constraints) to accurately define appropriate clusters that can safely unleash the required flexibility amounts (by the energy supplier for optimizing energy trading business functions, or by energy service companies (ESCOs) facility managers or aggregators for optimizing energy management in buildings and reducing energy costs of their customers, while optimizing the effectiveness of energy performance contracts), without compromising comfort, indoor environment quality or daily operations in buildings.

- District monitoring and control dispatch module which continuously monitors the evolution of the demand response event, to identify overrides of the deployed strategies or failures to respond and devises the initially defined strategies so as to achieve the provision of the anticipated amounts of flexibility and in this sense, optimizes business functions and energy transactions of all stakeholders involved.

In explicit demand response programmes, the DSS utilizes and process information received by the flexibility forecasting, segmentation and aggregation module and the district monitoring and control dispatch module for the selection of appropriate aggregated demand side setup to provide specific demand response functions enabling the provision of services for stable and secure grids (considering the flexibility profiles, along with the suitability of such profiles to provide different services to the grid, e.g. balancing, frequency response or voltage regulation). As, also described before, the global demand manager continuously monitors the progress of the demand response event and devise alternative strategies (deployment of additional flexibility sources) to safeguard the smooth and successful response to distribution system operator (DSO) requirements.

- The local demand manager. This component which is responsible for breaking down the global flexibility requirements into the flexibility that can be offered by each distinct load at the consumer side and for automatically dispatching the appropriate control signals/ actions over these specific loads (following a human-centric approach that preserves comfort and indoor quality), either upon approval of the consumer (in implicit demand response) or through direct load control (in explicit demand response). The DSS incorporates advanced intelligence features for analyzing and forecasting local context-aware demand flexibility at the level of individual devices (local flexibility analysis and forecasting component). It enables optimal decisionmaking considering not only loads' flexibility (mainly heating, ventilation, air conditioning (HVAC) and lighting), but also local generation output (photovoltaic, combined heat and power, etc.) and the flexibility offered by the thermal storage capabilities of buildings (thermal inertia) and available equipment, such as water heaters (utilizing the virtual thermal energy storage module), to define and dispatch highly effective personalized and 
context-aware local demand response control strategies (through the building monitoring and control dispatch component), for energy costs reduction, provision of services to the grid, as well as, promotion at the utmost level of self-consumption, within a comfort and indoor environmental quality (IEQ) preserving framework. The incorporated intelligence also allows for the continuous monitoring of the demand response strategy deployment and devises alternative strategies (introduction of additional loads, or slight regulation of comfort through more aggressive control of HVAC and lighting loads) as a means of hedging control overrides or failures to respond.

\section{Visualization platform and end-user toolkit layer}

This layer integrates and appropriately configures/ extends a variety of modules already developed and validated, to deliver a fully-fledged suite of services and user interfaces (web, mobile) to the end-users of the project, towards addressing their needs for information provision and analytics. It consists in a wide range of interfaces and multi-purpose dashboards that will utilize intuitive human machine interface (HMI) techniques and address the needs of requirements of:

- Consumers for increasing awareness and understanding on consumption patterns and flexibility potential, while enabling non-intrusive communication of demand response signal, personalized guidance and advice provision and automated control signal dispatch over selected loads. Moreover the application gives the opportunity to consumers to setup individual rules for the control of specific devices and align them with variable tariff levels, in order to avoid being charged with increased tariffs, by shedding the operation of noncritical loads when energy is expensive or shifting it at low-price periods.

- Aggregators for optimal portfolio management, flexibility clustering and demand response strategies implementation monitoring.

- Energy suppliers/ retailers for portfolio flexibility analysis and improved energy trading.

- Facility managers for improved building energy management and predictive maintenance.

- DSOs for improved demand forecasting, flexibility analysis and demand response triggering.

\section{VALIDATION FRAMEWORK}

The solution is being evaluated over a period of twelve months and validated in real-life conditions by a large and diverse population of residential and commercial consumers in four dispersed geographical areas. Building occupants, energy retailers, aggregators and facility managers continuously remain at the heart of design, development and validation activities.

The selected validation sites exhibit diverse characteristics (climatic, cultural and demographic) to ensure a representative validation process, while ensuring a high replication potential around the European Union.
Finally, the scale of the prominent pilots, the diversity of involved stakeholders and the population size actively involved establish the necessary critical mass upon which the large-scale promotion and uptake of the solution is pursued.

The following tables summarize the key aspects and characteristics of the pilot sites:

TABLE I. CHARACTERISTICS OF VALIDATION SITES

\begin{tabular}{|l|c|c|c|c|}
\hline \multirow{2}{*}{ Characteristics } & \multicolumn{4}{|c|}{ Location } \\
\cline { 2 - 5 } & Athens, Greece & $\begin{array}{c}\text { London, } \\
\text { UK }\end{array}$ & $\begin{array}{c}\text { Helsinki, } \\
\text { Finland }\end{array}$ & $\begin{array}{c}\text { Belgrade, } \\
\text { Serbia }\end{array}$ \\
\hline Climate $^{\mathrm{a}}$ & $\begin{array}{c}\text { Mediterranean } \\
\text { North }\end{array}$ & $\begin{array}{c}\text { Atlantic } \\
\text { North }\end{array}$ & Boreal & $\begin{array}{c}\text { Pannonic/ } \\
\text { Pontic }\end{array}$ \\
\hline $\begin{array}{l}\text { Number and } \\
\text { type of } \\
\text { buildings }\end{array}$ & $\begin{array}{c}2 \mathrm{com}^{\mathrm{b}} \\
53 \mathrm{dwe}^{\mathrm{c}}\end{array}$ & $\begin{array}{c}3 \mathrm{com} \\
170 \mathrm{dwe}\end{array}$ & $\begin{array}{c}2 \mathrm{com} \\
188 \mathrm{dwe}\end{array}$ & $\begin{array}{c}2 \mathrm{com} \\
3,500 \mathrm{dwe}\end{array}$ \\
\hline $\begin{array}{l}\text { Total surface } \\
\text { [m }^{2} \text { ] }\end{array}$ & 17,112 & 83,500 & 66,300 & 170,000 \\
\hline $\begin{array}{l}\text { Annual electric } \\
\text { consum.[MWh] }\end{array}$ & 1,960 & 21,586 & 5,532 & 8,660 \\
\hline $\begin{array}{l}\text { Annual gas } \\
\text { consum.[MWh] }\end{array}$ & - & 15,570 & - & - \\
\hline $\begin{array}{l}\text { Annual DH } \\
\text { consum.[MWh] } \\
\text { consum }\end{array}$ & - & - & 4,173 & 3,890 \\
\hline $\begin{array}{l}\text { Total annual } \\
\text { energy costs [€] }\end{array}$ & 154,180 & $3,902,650$ & 677,850 & $2,308,250$ \\
\hline
\end{tabular}

a. European Environmental Stratification [7], ${ }^{\text {b. }}$ commercial, ${ }^{\mathrm{c}}$ dwellings, ${ }^{\mathrm{d} .}$ district heating

TABLE II. TECHNOLOGIES ON VALIDATION SITES

\begin{tabular}{|l|c|c|c|c|}
\hline \multirow{2}{*}{$\begin{array}{c}\text { Technologies } \\
\text { deployed }\end{array}$} & \multicolumn{5}{c|}{ Location } \\
\cline { 2 - 5 } & $\begin{array}{c}\text { Athens, } \\
\text { Greece }\end{array}$ & $\begin{array}{c}\text { London, } \\
\text { UK }\end{array}$ & $\begin{array}{c}\text { Helsinki, } \\
\text { Finland }\end{array}$ & $\begin{array}{c}\text { Belgrade, } \\
\text { Serbia }\end{array}$ \\
\hline Smart metering & $\mathrm{x}$ & $\mathrm{x}$ & $\mathrm{x}$ & $\mathrm{x}$ \\
\hline Smart thermostats & $\mathrm{x}$ & $\mathrm{x}$ & $\mathrm{x}$ & $\mathrm{x}$ \\
\hline Smart Lighting & $\mathrm{x}$ & $\mathrm{x}$ & $\mathrm{x}$ & $\mathrm{x}$ \\
\hline Sensors & $\mathrm{x}$ & $\mathrm{x}$ & & $\mathrm{x}$ \\
\hline Smart home EMS & $\mathrm{x}$ & $\mathrm{x}$ & $\mathrm{x}$ & $\mathrm{x}$ \\
\hline Thermal storage & $\mathrm{x}$ & $\mathrm{x}$ & & $\mathrm{x}$ \\
\hline Photovoltaics & & $\mathrm{x}$ & $\mathrm{x}$ & \\
\hline Backup generators & & $\mathrm{x}$ & & \\
\hline
\end{tabular}

In order to facilitate the cross-site assessment of the performance, transferability and scalability of the framework, pilot demonstrations are conducted following the eight high level use cases (UC) listed below:

- UC-1: Personalized, flexibility-based guidance of individual consumers for optimizing their participation in implicit demand response schemes and significantly reducing their energy bills through real-time pricing and its combination with critical peak pricing and peak time rebates schemes.

- UC-2: Promotion of net metering and self-consumption through holistic optimization of demand, supply and storage at the building and district level. 
- UC-3: Context-aware demand flexibility profiling against volatile and variable real-time energy prices, considering user preferences for comfort, IEQ and daily schedules.

- UC-4: Personalized, human-centric and contractsafeguarding participation in explicit demand response programmes, on the basis of context-aware flexibility profiles and with the aim to increase consumer benefits through aggregated bidding of flexibility in balancing and ancillary services markets.

- UC-5: End-to-end interoperability (independently of brands, products and communication protocols) between actors, energy management systems and field-area devices.

- UC-6: Decision support to energy suppliers (and third parties acting as energy service providers, e.g. aggregators, ESCOs and facility managers) for optimal segmentation, clustering, e.g. virtual power plants (VPP) and utilization of demand side flexibility for energy trading and energy management optimization.

- UC-7: Decision support to aggregators (and third parties acting as aggregators, e.g. energy suppliers, ESCOs and facility managers) for optimal segmentation, clustering (VPP) and utilization of demand side flexibility for a provision of a variety of services to the grid.

- UC-8: Predictive maintenance of critical building equipment for improving energy performance, avoiding unnecessary energy consumption and improving comfort and IEQ.

The following table presents the alignment of the aforementioned high-level use cases with the demonstration activities to be performed in the four pilot sites:

TABLE III. USE CASES IN PILOT SITES

\begin{tabular}{|l|c|c|c|c|}
\hline \multirow{2}{*}{ UC/Pilot site } & \multicolumn{4}{|c|}{ Location } \\
\cline { 2 - 5 } & $\begin{array}{c}\text { Athens, } \\
\text { Greece }\end{array}$ & $\begin{array}{c}\text { London, } \\
\text { United } \\
\text { Kingdom }\end{array}$ & $\begin{array}{c}\text { Helsinki, } \\
\text { Finland }\end{array}$ & $\begin{array}{c}\text { Belgrade, } \\
\text { Serbia }\end{array}$ \\
\hline UC-1 & $\mathrm{x}$ & $\mathrm{x}$ & $\mathrm{x}$ & $\mathrm{x}$ \\
\hline UC-2 & $\mathrm{x}$ & $\mathrm{x}$ & $\mathrm{x}$ & \\
\hline UC-3 & $\mathrm{x}$ & & & $\mathrm{x}$ \\
\hline UC-4 & $\mathrm{x}$ & $\mathrm{x}$ & $\mathrm{x}$ & $\mathrm{x}$ \\
\hline UC-5 & $\mathrm{x}$ & $\mathrm{x}$ & $\mathrm{x}$ & $\mathrm{x}$ \\
\hline UC-6 & & $\mathrm{x}$ & & \\
\hline UC-7 & & & $\mathrm{x}$ & \\
\hline UC-8 & & &
\end{tabular}

\section{CONCLUSIONS}

The integration of demand response enabling elements into EMS towards optimizing, at building level, energy consumption, production and storage considering the availability and price of energy supplied via the grid is a challenge. A specific challenge is that EMS and smart home devices are often not interoperable but are linked to a certain brand, technology and/or standard. Full interoperability between grids, systems and products for seamless integration of all required components in building energy management systems is crucial.

This paper presents a novel solution that introduces residential and tertiary energy consumers as active players in energy markets and ensure significant benefits through their engagement in implicit human-centric demand response programmes. Also it enables intelligence enhancement of currently available BEMS and smart home systems with the integration of information and communication technologiesenabled human-centric demand response optimization and predictive maintenance functions.

Furthermore the solution delivers an open standards-based modular solution that ensures end-to-end interoperability between smart grids, EMS and smart home devices and holds a high replication potential around European Union Member States. It safeguards grid reliability and the transition to a more fossil-free energy future through complementary explicit demand response strategies on the basis of aggregated flexibility utilization. It tackles major market entry barriers for consumers with the introduction of suitable business models for energy utilities, aggregators and facility managers.

Finally the adoption of the solution as a next-generation demand response optimization framework is promoted by validating the resulting solutions in real-life environments and ensure enhanced consumer engagement in demand response.

\section{REFERENCES}

[1] Smart Energy Demand Coalition, "Empowering Residential and SME Consumers," 2016.

[2] J. Stromback, "Status of Demand Response in Europe," Smart Energy Demand Coalition, 2015.

[3] European Commision, "Commission Staff Working Document: Energy prices and costs report," 2014.

[4] ACER, "Wholesale gas prices continue to fall in the EU but electricity prices end their decreasing trend in some countries," 2016.

[5] European Commission, "Progress towards completing the Internal Energy Market," 2014.

[6] European Commision, "Clean Energy For All Europeans," COM(2016) 860 final.

[7] M.J. Metzger, R. G. H. Bunce, R. H. G. Jongman, C. A. Mücher, and J. W.Watkins, "A climatic stratification of the environment of Europe," Global Ecology and Biogeography, vol. 14, pp.549-563, Nov 2005. 